Faculté des lettres, Université Charles, Prague

DOI: 10.4312/vestnik.9.191-200

Institut National des Langues et

Civilisations Orientales (INALCO),

Laboratoire PLIDAM

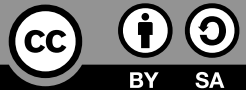

gregoire.labbe@ff.cuni.cz

\title{
SUR QUELLES BASES ENSEIGNER \\ L'INTERCOMPRÉHENSION ENTRE LES LANGUES SLAVES DE L'OUEST ET DU SUD-OUEST?
}

\section{Introduction}

Bien des locuteurs natifs d'une langue romane, germanique ou slave l'ont ressenti lors d'un voyage, du visionnage d'un film, de la lecture d'un panneau d'informations ou dans le cadre d'une autre situation les confrontant à une langue de la même famille: la compréhension de ce qui est dit ou écrit est là, presque accessible, à portée de main. Il ne manquerait pas grand-chose pour qu'elle soit totale, mais certains mots, certaines formes, structures et sonorités font obstacle. C'est sur ce constat que se sont basées les premières théories visant au développement de concepts pédagogiques autour de l'enseignement de l'intercompréhension entre les langues romanes (Dabène, 1996 ; Blanche-Benveniste, 1997). Aujourd'hui, la variété des approches pédagogiques en la matière est remarquable et l'enseignement de l'intercompréhension est un domaine en plein essor qui gagnerait à se généraliser dans de nombreuses familles de langues. Les langues slaves semblent pour cela toutes désignées.

Parmi les projets aboutis et disponibles à ce jour, nous pouvons citer, principalement, les ouvrages Slavische Interkomprehension - Eine Einfuehrung (Tafel, 2009) et Slawischer Sprachvergleich für die Praxis (Heinz et Kuße, 2015). Ces deux ouvrages ont comme point commun de présenter cinq langues slaves choisies (russe, ukrainien, polonais, tchèque et BCMS pour le premier et russe, polonais, tchèque, BCMS et bulgare pour le second) dans les contextes diachroniques et synchroniques de la famille slave, de façon comparée. Par BCMS, on entendra ici les variantes bosnienne, croate, monténégrine et serbe d'une même langue, autrefois appelée serbo-croate.

Ces deux ouvrages sont destinés à un public d'apprenants germanophones. L'allemand y est utilisé comme langue d'enseignement et le système slave est abordé de façon «neutre», «par le haut», sans imposer de langue pont en particulier. 


\section{UNE GRANDE VARIÉTÉ DE CONCEPTS - L'EXEMPLE DE LA FAMILLE DES LANGUES ROMANES}

Il estégalement intéressant de se pencher sur les méthodes consacrées aux langues romanes pour comprendre à quels publics peut s'adresser l'enseignement de l'intercompréhension:

- Comprendre les langues romanes (Teyssier, 2003) présente le système linguistique roman à travers le français, le portugais, l'espagnol, l'italien et le roumain. Il est rédigé en français et est donc destiné à un public francophone connaisseur du lexique et de la grammaire française, expliquant et complétant le système roman de façon ciblée, par rapport aux besoins d'un francophone.

- Eurom 5 (Bonvino et al., 2011) est accessible depuis n'importe laquelle des langues sur lesquelles la méthode est fondée (portugais, espagnol, catalan, italien, français). La structure de la méthode est pensée de façon à ce que chacune de ces langues puisse faire office de langue pont vers la compréhension du système roman, n'apportant à l'apprenant que les connaissances liées aux langues qu'il ne connaît pas. Il n'est pas obligatoire que l'apprenant soit un locuteur natif de l'une de ces langues, les connaissances apportées par un apprentissage de qualité d'une de ces langues peuvent suffire.

- EuroComRom (Meissner, F-J et al., 2004) est destiné à un public germanophone ayant une bonne connaissance du français. La méthode est conçue sur le constat que la langue romane la plus étudiée en Allemagne est le français et fournit aux apprenants les informations complémentaires pour comprendre le système roman, en se concentrant sur les langues suivantes: portugais, espagnol, catalan, italien et roumain. Une version slave de cette méthode était prévue, elle est toutefois restée inachevée et n'est à ce jour plus disponible. La méthodologie EuroCom a dans une grande mesure inspiré les travaux en intercompréhension slave réalisés dans les ouvrages Slavische Interkomprehension - Eine Einfuehrung et Slawischer Sprachvergleich für die Praxis brièvement présentés un peu plus tôt.

- Le manuel scolaire Euromania (Escudé et al., 2008) est destiné à un public d'enfants ayant l'âge d'aller à l'école primaire. Il est disponible dans plusieurs langues, selon la langue maternelle des enfants concernés. Il propose une initiation à l'intercompréhension, sans toutefois chercher à fournir une compréhension approfondie de chaque langue. Il s'agit plutôt d'un premier contact visant à stimuler la curiosité, à montrer simplement et de façon ludique la proximité des langues romanes et à réduire l'angoisse liée à l'apprentissage d'une langue.

\section{OBJECTIFS ET PUBLICS CONCERNÉS PAR CES MÉTHODES.}

La pédagogie de l'intercompréhension peut donc avoir autant de formes que de publics et il serait donc erroné de se la représenter de façon uniforme. Il est essentiel de se 
poser la question de l'objectif visé et du public concerné avant de penser à la forme de la méthode.

Les méthodes citées ci-dessus visent l'un des deux objectifs suivants:

- Apprendre à comprendre les langues d'une famille lorsque l'on en maîtrise déjà une (Eurom5, EuroComRom, Slawischer Sprachvergleich für die Praxis...)

- Se sensibiliser à la compréhension des langues d'une même famille (Euromania)

Nous laisserons ce second objectif de côté pour nous intéresser de plus près à l'apprentissage de la compréhension.

L'autre critère important est celui du public concerné, notamment au niveau de la (ou des) langue(s) utilisée(s) comme porte(s) d'entrée vers l'intercompréhension. Nous tenterons de résumer les types d'approches possibles par rapport aux publics concernés de la façon suivante, sans toutefois prétendre à une exhaustivité absolue:

1. L'approche peut être destinée aux locuteurs d'une langue particulière, se contentant de fournir des informations ciblées permettant l'accès à une bonne compréhension des autres langues de cette famille. Ce type d'approche est intuitif et permet une acquisition plus rapide des connaissances. Le principal inconvénient est l'impossibilité d'adapter cette approche à un locuteur d'une autre langue, même proche. La méthode EuroComRom est, à l'origine, conçue de cette façon: l'apprenant doit être germanophone et avoir appris le français. Une connaissance de l'espagnol à la place du français nuirait à son efficacité.

2. Cette approche peut toutefois être multipliée par le nombre de langues auxquelles se consacre la méthode: Dans Eurom5, n'importe laquelle des langues abordées peut servir de langue pont. Toutes les explications sont disponibles dans les 5 langues, les locuteurs d'une langue peuvent ignorer les leçons portant sur celle-ci et dans tous les cas, la connaissance du système roman n'est complétée que par des connaissances « utiles ». L'avantage de cette approche est bien sûr sa souplesse, mais il est évident qu'un travail bien plus approfondi est nécessaire pour arriver à un tel résultat.

3. Une autre façon d'aborder l'enseignement de l'intercompréhension est de ne pas favoriser de langue en particulier et de présenter le système d'une famille dans son intégralité, montrant aussi bien les règles inhérentes au système et les exceptions propres à chaque langue. C'est ce que les ouvrages Slavische Interkomprehension Eine Einfuehrung et Slawischer Sprachvergleich für die Praxis proposent. Ils partent du principe qu'une des langues abordées est mieux connue par l'apprenant, mais ne font pas d'hypothèse sur la langue en question. Ils présentent de façon uniforme chacune d'entre elles. Une telle neutralité est garantie par le choix d'une langue d'enseignement extérieure à la famille de langues étudiée: dans ce cas, il s'agit de l'allemand. Dans le cas où la langue d'enseignement serait l'une des langues de la famille étudiée, nous nous retrouverions en présence d'un ouvrage comparable à Comprendre les langues romanes, rédigé en français et partant donc du principe que 
le lecteur a plutôt besoin de compléter ses connaissances autour de ce qu'il connaît déjà du système linguistique roman grâce au français. Dans un cas comme dans l'autre, le public ciblé est limité par la langue d'enseignement choisie.

Résumer le public ciblé par rapport à la langue pont et à la langue d'enseignement utilisée serait réducteur. Il est aussi nécessaire de prendre en considération d'autres paramètres, tels que le degré de spécialisation:

Les approches 1 et 2, plus ciblées sur les besoins de l'apprenant, rendent possible une méthodologie plus adaptée à un public non linguiste et simplement désireux de comprendre sans grande difficulté les autres langues d'une famille, sans toutefois faire l'impasse sur certains points du système linguistique.

L'approche 3, plus neutre, est sûrement plus adaptée à un public de linguistes soucieux d'avoir en tête la structure la plus objective possible du système de la famille de langues concernée.

Les approches 1 et 2 laisseraient donc en cela plus de place à des pédagogies originales telle que celle proposée par la méthode Eurom 5.

\section{$4 \quad$ EXEMPLE DE MÉTHODOLOGIE: LE CAS D’EUROM 5}

- Tel que nous l'avons mentionné, Eurom 5 propose une pédagogie basée sur cinq langues: le portugais, l'espagnol, le catalan, l'italien et le français et succède à Eurom 4, créé et dirigé par Claire Blanche-Benveniste. La méthode peut être abordée par quiconque possédant déjà une bonne maîtrise de l'une de ces langues. Une pédagogie originale basée sur des articles de journaux confronte directement l'apprenant à la lecture. Dans le cadre d'une leçon, il devra lire un article dans chacune des langues qu'il ne connaît pas. La leçon adaptée au cas d'un français ne parlant pas d'autres langues romanes commencera par la lecture de l'article portugais, puis des articles espagnol, catalan et italien. L'ordre est important et suit au mieux le continuum linguistique.

La lecture d'un article se fait toujours comme suit:

Le texte nu est survolé une première fois de façon à ne pas s'arrêter sur les différences. Le but est au contraire de le lire rapidement et avec fluidité, comme s'il s'agissait d'un texte rédigé dans notre langue maternelle. Ainsi, notre œil ne retiendra que ce qui lui est familier. A l'issue de cette première lecture, le thème ainsi que certaines informations du texte sont généralement compris. La seule aide fournie est une traduction du titre de l'article dans les 4 autres langues romanes. Nous prendrons soin de lire les traductions dans chacune des langues auxquelles nous nous initions, en respectant le continuum linguistique, avant de lire la traduction fournie dans la langue connue. Ce faisant, nous 
avons souvent possibilité d'en comprendre le sens avant d'arriver à notre propre langue, et certaines équivalences se créent.

La seconde étape est l'écoute de la version audio de l'article. Cette écoute stimule la compréhension orale, permet d'associer une sonorité à une orthographe et peut faciliter la compréhension du sens d'un ou plusieurs mots. En effet, il peut arriver qu'une orthographe opaque corresponde à une sonorité connue (pour un Français ne connaissant rien du portugais, il pourra être difficile de faire le lien entre l'orthographe portugaise «ã̃» et la nasale française «on», alors qu'il s'agit à l'oral du même son).

La troisième étape est une relecture de l'article accompagnée du minimum d'annotations et d'aides nécessaires à sa bonne compréhension. Nous aurons ainsi accès à des traductions là où le sens d'un mot ne peut pas être déduit d'une autre manière, à des points de grammaire comparée là où le système grammatical d'une langue donnée diffère par rapport à d'autres ou encore à des aides syntaxiques permettant de décrypter des structures compliquées. Il peut par exemple être difficile à un Français d'identifier un sujet non exprimé. En effet, alors que la présence d'un sujet est généralement obligatoire en français, le pronom personnel peut ne pas apparaître dans les autres langues romanes.

Nous avons donc, avec Eurom 5, un concept pédagogique complet et original très éloigné de l'apprentissage chapitre par chapitre de points de grammaire comparée qui pourrait rebuter un public non spécialisé. Les articles choisis, aux thématiques souvent curieuses voire amusantes, stimulent la motivation de l'apprenant à progresser et à comprendre.

\section{EXPÉRIENCE D'ADAPTATION DU CONCEPT D'EUROM 5 À UN PETIT CHOIX DE LANGUES SLAVES}

Sur ce constat, nous avons souhaité proposer aux étudiants tchécophones de l'université Charles de Prague une initiation d'un semestre à la compréhension du slovène et, dans une moindre mesure, du croate, largement inspirée dans les grandes lignes par la méthode Eurom 5 et qui en respecte la structure.

Le public concerné est donc un public tchécophone désireux de comprendre le slovène. Chaque leçon est centrée soit sur un article de journal complété par un enregistrement vocal effectué par un locuteur natif, soit sur un document sonore complété par une transcription prenant en compte les éventuelles formes parlées, tics de langages, particulièrement utiles à la compréhension orale du slovène. Le croate n'est ici utilisé que de façon complémentaire, dans les listes de vocabulaire ou les explications d'ordre grammatical, afin de fournir à l'apprenant un meilleur aperçu des possibilités qu'offrent les langues slaves du sud-ouest.

L'objectif de cette expérience a tout d'abord été de déterminer les besoins spécifiques d'un locuteur de tchèque qui souhaiterait mieux comprendre le slovène. L'expérience 
s'est déroulée sur deux sessions d'un semestre sur l'année universitaire 2016-2017, la $2^{\mathrm{e}}$ session ayant connu tout un lot d'ajustements permettant de mieux cibler les besoins des apprenants. Les différences principales entre les deux sessions sont les suivantes:

- Ajustements d'ordre grammatical: meilleur ciblage sur les points de grammaire qui font obstacle à la compréhension.

- Ajustements d'ordre lexical: utilisation de documents contenant plus de faux amis problématiques. CZ: zapomenout $>$ oublier $\quad$ SI: zapomniti si $>$ se souvenir

- Plus grande attention portée aux documents sonores. C'est en effet à partir de la $2^{\mathrm{e}}$ session que certaines leçons partent d'un document sonore pour ne s'attarder qu'en second lieu sur la transcription. La $1^{\text {ère }}$ session ne comportait que des leçons classiques partant d'articles de journaux complétés par des enregistrements. Cela permet à l'apprenant d'être mieux initié à la compréhension orale, plus difficile à acquérir que la compréhension écrite. De plus, l'une des grandes difficultés du slovène par rapport au tchèque est d'ordre phonétique, pour plusieurs raisons:

- Accent mobile difficilement prévisible en slovène et accent fixe en début de mot en tchèque;

- Grandes différences de prononciation entre les locuteurs slovènes selon leur région d'origine;

- Orthographe n'apportant pas toutes les informations nécessaires à la bonne prononciation.

Un même test de compréhension écrite est distribué en début et en fin de session. Il permet de mieux appréhender l'évolution de la compréhension sur les difficultés du slovène. L'apprenant y a pour tâche d'expliquer plusieurs phrases les reflétant au mieux. Une fois rempli une première fois lors de la séance d'introduction, le test est conservé en l'état et ne fait l'objet d'aucun commentaire. Lors du second remplissage, après la dernière séance, l'apprenant est censé être en mesure de reconnaître, dans chacune de ces phrases, les points abordés tout au long du semestre et ainsi de comprendre ces phrases dans leur globalité.

Po dolgem čakanju in v tej vročini ni lahko aktivno sodelovati.

$>$ Après une longue attente dans cette chaleur, il n'est pas facile de coopérer activement.

Lahko utišaš glasbo? > Tu peux baisser la musique?

Les deux phrases ci-dessus contiennent le mot « lahko », qu'un apprenant tchèque aura naturellement tendance, à raison, à associer avec l'adjectif « lehký » (facile, léger). C'est d'ailleurs dans ce sens que la $1^{\text {ère }}$ phrase l'utilise. En revanche, la $2^{\text {nde }}$ phrase utilise l'adverbe « lahko » d'une façon tout à fait spécifique au slovène. La structure «lahko »+ verbe 
conjugué est une structure modale équivalente au verbe « pouvoir » suivi de l'infinitif. Le tchèque fonctionne ici comme le français avec le verbe « moci » suivi de l'infinitif. Un apprenant tchèque aura tendance à traduire la $2^{\mathrm{e}}$ phrase « tu baisses un peu la musique ? », ce qui n'est pas ici un contresens total, mais traduit clairement l'incapacité à reconnaître la structure modale. Il est également évident qu'il suffira d'évoquer rapidement une règle simple pour que l'apprenant sache une fois pour toutes comment interpréter cette structure.

Un fichier contenant toutes les notes grammaticales utilisées lors de la session est distribué lors de la $1^{\text {ère }}$ véritable séance de compréhension. Tout comme dans la méthode Eurom 5, l'apprenant peut s'y référer autant que nécessaire grâce à un système d'annotations des textes étudiés. Ainsi, à chaque fois que la structure «lahko» + verbe conjugué est utilisée, la référence du point de grammaire correspondant est indiquée en marge du texte, laissant à l'apprenant le loisir de s'y référer tant qu'il aura des doutes concernant cette règle.

$\mathrm{Au}$ niveau lexical, une attention toute particulière est portée sur les mots de liaison, essentiels à la bonne compréhension de la structure de la phrase et particulièrement faciles à confondre lorsque l'on passe d'une langue à l'autre, comme le montrent les exemples suivants:
- $\quad$ SI: ali (ou)
CZ: ale (mais)
- $\quad$ SI: ampak (mais)
CZ: a pak (et ensuite)

Une confrontation aux principaux faux amis est également assurée par les textes étudiés lors de la session d'intercompréhension, car il s'agit de mots que l'apprenant n'aurait souvent pas idée de chercher dans le dictionnaire, altérant ainsi le sens des documents lus.

En revanche, il est à noter que le gros de la flexion en slovène ne représente aucun obstacle de compréhension pour un tchécophone, ce qui montre à quel point l'approche ciblée apporte de gain de temps et d'efficacité en permettant de se passer de tableaux de déclinaison et de conjugaison.

A l'issue des deux sessions réalisées à ce jour, l'évolution de la compréhension observée chez les apprenants est sans équivoque et est confirmée par les résultats positifs aux tests de fin de session. Ces résultats sont toutefois à nuancer, dans la mesure où le nombre d'apprenants testé est faible (2 par sessions).

\section{6}

\section{CONCLUSION}

Notre étude ne cherche pas à démontrer qu'un enseignement de l'intercompréhension entre les langues slaves est possible et potentiellement accessible à tous. En effet, nous partons du principe que cela est déjà démontré, ne serait-ce que par la multitude de méthodes efficaces consacrées à la pédagogie de l'intercompréhension entre les langues 
romanes et par les multiples travaux réalisés en matière de slavistique. Notre but est au contraire de profiter de l'expérience romane de l'enseignement de l'intercompréhension afin de proposer une approche parmi de nombreuses autres possibles adaptées aux langues slaves. Nous sommes convaincu de la grande utilité de telles approches, d'autant plus que les langues de l'Union Européenne sont, dans leur grande majorité, issues de trois grandes familles particulièrement propices à l'intercompréhension (les langues romanes, slaves et germaniques). Un tel apprentissage n'est pas forcément long ou compliqué à mettre en place et peut constituer un plus non négligeable à l'apprentissage des langues classiquement enseignées telles que l'anglais.

\section{RÉFÉRENCES BIBLIOGRAPHIQUES :}

BLANCHE-BENVENISTE, Claire (1997) «L'intercompréhension: le cas des langues romanes», Le français dans le monde, $\mathrm{n}^{\circ} 11,5-7$.

BONVINO, Elisabetta/Sandrine CADDEO/Salvadore PIPPA (2011) Eurom 5, Lire et comprendre 5 langues romanes. Milan, Hoepli, 554.

BONVINO, Elisabetta/Elisa FIORENZA/Salvador PIPPA (2011) «EuRom5, una metodologia per l'intercomprensione. Strategie, aspetti linguistici e applicazioni pratiche», Maddalena De Carlo (dir.), Intercomprensione e educazione al plurilinguismo. Porto, Wizarts, 162-182.

CADDEO, Sandrine/Marie-Christine JAMET (2013) L'intercompréhension: une autre approche pour l'enseignement des langues. Paris, Hachette «collection F», 160.

DABÈNE, Louise (1996) «Présentation», Etudes de Linguistique Appliquée, n¹04, 389-391.

ESCUDÉ, Pierre et al. (2008) Euromania. Toulouse, IUFM Midi-Pyrénées, 20 fascicules de $8 \mathrm{p}$.

ESCUDÉ, Pierre/Pierre JANIN (2010) Le point sur l'intercompréhension, clé du plurilinguisme, Paris, CLE International, 122.

HEINZ, Christof/Holger KUSSE (2015) Slawischer Sprachvergleich für die Praxis. Munich, Berlin, Leipzig, Washington, BiblionMedia «Specimina Philologiae Slavicae», 462.

KLEIN, Horst G. (2002)《EuroCom - Europäische Interkomprehension», Dorothea Rutke (dir.), Europäische Mehrsprachigkeit: Analysen - Konzepte - Dokumente, Aix-la-Chapelle, Shaker, 29-44.

KLEIN, Horst G. (2004) «L'Eurocompréhension (EuroCom), une méthode de compréhension des langues voisines», Etudes de Linguistique Appliquée n ${ }^{\circ}$ 136, 403-418.

MEISSNER, Franz-Josef/Claude MEISSNER/Horst G. KLEIN/Tilbert D. STEGMANN (2004) EuroComRom - Les sept tamis: lire les langues romanes dès le départ ; avec une esquisse de la didactique de l'eurocompréhension. Aix-la-Chapelle, Shaker, 336. 
TAFEL, Karin (2009) Slavische Interkomprehension: Eine Einführung, Tübingen, Gunter Narr, «Narr Studienbücher», 267.

TEYSSIER, Paul (2004) Comprendre les langues romanes. Du français à l'espagnol, au portugais, à l'italien et au roumain. Méthode d'intercompréhension. Paris, Chandeigne, 396.

\section{POVZETEK}

Na kakšni osnovi se lahko poučuje medjezikovno razumevanje med zahodnimi in jugozahodnimi slovanskimi jeziki?

Medjezikovno razumevanje med podobnimi jeziki, ki pripadajo isti jezikovni skupini je, do neke mere, naravni pojav. Vendar pa je razumevanje bližnjih jezikov pogosto moteno zaradi mnogih nejasnih elementov, ki so nerazumljivi brez poznavanja slovnice ali besedišča jezika. Metodologija, podobna tisti, ki jo ponuja priročnik Eurom 5, dopušča učencem izboljšanje poznavanja jezikovne skupine in njenega delovanja. V priročniku Eurom 5 se učni proces osredotoča na poznavanje »romanskega sistema« in izpostavlja podrobnosti posameznih jezikov, ki jih priročnik obravnava. Takšen sistem zagotavlja boljše razumevanje teh jezikov. Koncept smo poskušali prirediti za zahodne in jugozahodne slovanske jezike, s poudarkom na češčini, slovenščini in, do manjše mere, hrvaščini.

Ključne besede: medjezikovno razumevanje, poučevanje, romanski jeziki, slovanski jeziki, slovenščina, češčina, hrvaščina

\section{ABSTRACT}

On what basis is it possible to teach intercomprehension between western and south-western Slavic languages?

Intercomprehension between similar languages of the same family is to a certain measure a natural phenomenon. However, comprehension of a close language will very often be disturbed by many opaque elements, which are not understandable without a better knowledge of its grammar or vocabulary. A methodology similar to the one offered by the manual Eurom 5 allows learners to work on their comprehension of a language family and its functioning. In Eurom 5, the learning process is focused on the knowledge of a "romance system", bringing out the particularities of each of the languages dealt with within the manual. With such a system in place, a better comprehension of these languages is guaranteed. We have been trying to adapt such a concept to western 
and south-western Slavic languages, focusing at first on Czech, Slovenian and, to a lesser extent, Croatian.

Key words: intercomprehension, teaching, Romance languages, Slavic languages, Slovenian, Czech, Croatian

\section{RÉSUMÉ}

L'intercompréhension entre les langues proches d'une même famille est un phénomène en grande partie naturel. Cependant, le plus souvent, la compréhension d'une langue proche sera gênée par de nombreux éléments opaques que seule une meilleure connaissance de cette langue peut éclaircir. Ce que propose une méthode telle qu'Eurom 5, c'est un travail permettant de faire connaissance avec une famille de langues et son fonctionnement (ici les langues romanes), un « système roman » mettant en évidence les particularités de chaque langue traitée pour en garantir une bonne compréhension. Nous avons tenté d'adapter un tel concept aux langues slaves d'Europe de l'ouest et du sud-ouest, en nous concentrant, dans un premier temps, sur le tchèque, le slovène et, dans une moindre mesure, le croate.

Mots-clés: intercompréhension, enseignement, langues romanes, langues slaves, slovène, tchèque, croate 